\title{
Agricultura de conservación de suelos y su efecto en la erosión hídrica y propiedades hidrofísicas en la unidad hidrográfica Quebrada Arriba, Yalagüina, 2017
}

\section{Soil conservation agriculture and its effect on water erosion and hydrophysical properties in the Quebrada Arriba hydrographic unit, Yalagüina, 2017}

\author{
Gabriel Martin Llanes ${ }^{1}$, Darwin Omar Rizo Bermúdez¹, Reynaldo Bismarck Mendoza Corrales², Esteban Antonio \\ Avilés Silva ${ }^{2}$, Henry Alberto Duarte Canales ${ }^{3}$ \\ ${ }^{1}$ Ingeniero Agrícola graduado de la Universidad Nacional Agraria, ORCID: https://orcid.org/0000-0002-8984-3295 - gllanes51@gmail.com \\ / ORCID: https://orcid.org/0000-0003-2121-1942 - bdarwin131@gmail.com \\ ${ }^{2}$ MSc. Docente investigador Facultad de Recurso Naturales y el Ambiente, ORCID: https://orcid.org/0000-0003-4382-7983 - rmendoza@ \\ ci.una.edu.ni / ORCID: https://orcid.org/0000-0003-3936-6811 - aaviles@ci.una.edu.ni \\ ${ }^{3}$ MSc. Docente investigador Facultad de Agronomía, ORCID: https://orcid.org/0000-0003-3515-845X - hduarte@ci.una.edu.ni \\ Universidad Nacional Agraria
}

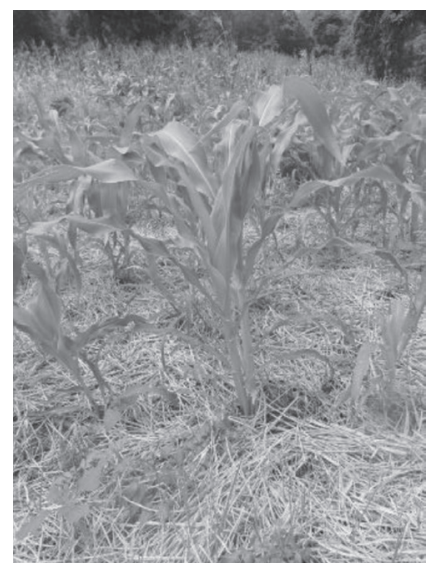

\section{RESUMEN}

Con el propósito de evaluar el efecto de los usos de suelo; sistema de coberturas de suelo (SCS), agroforestal (SAF), tradicional (Tr) y bosque secundario (Bs) sobre la erosión hídrica y la restauración de las propiedades hidrofísicas; infiltración del agua en el suelo, contenidos de arcilla, materia orgánica, y porcentaje de humedad a capacidad de campo. Fue establecido un ensayo experimental de parcelas divididas en tres fincas localizadas la unidad hidrográfica Quebrada Arriba, Yalagüina-Madriz, Nicaragua. Los sistemas que presentan mayor cobertura superficial presentaron, menor tasa de erosión de 41.75 ton ha ${ }^{-1}$ en los SAF versus 123.08 ton ha $^{-1}$ en los sistemas tradicionales. Y mayores contenidos de materia orgánica de $10 \%$ en el sistema ASA a $2.98 \%$ en el tradicional. A nivel espacial los tratamientos que se ubicaron en la parte baja del relieve, presentaron mayores porcentajes de humedad retenida y materia orgánica (MOS) en comparación a los tratamientos ubicados en la parte alta. Finalmente, los altos contenidos de arcilla contribuyen al incremento de la capacidad de retener agua en el suelo de la microcuenca. Por tanto, es necesario promover tecnologías que brinden al suelo mayor cobertura para mejorar su calidad y reducir la tasa erosión hídrica.

Palabras clave: retención de humedad, varillas de erosión, laderas, corredor seco.

\begin{abstract}
In order to evaluating the effect of land uses, soil cover systems (SCS), agroforestry (SAF), traditional (Tr) and secondary forest (Bs) on water erosion and restoration of soil hydrophysical properties. Soil water infiltration, clay contents, soil organic matter, and the percentage of humidity at field capacity. An experimental trial of divided plots was established in three farms located in the Quebrada Arriba hydrographic unit, Yalagüina-Madriz, Nicaragua. The systems with the greatest surface coverage showed a lower erosion rate of 41.75 tons per ha ${ }^{-1}$ in the SAF versus 123.08 tons per ha ${ }^{-1}$ in the traditional systems. Moreover, higher organic matter contents from $10 \%$ in the ASA system to $2.98 \%$ in the traditional one. At the spatial level the treatments that were located in the lower part of the relief, presented higher percentages of retained moisture and organic matter (MOS) compared to the treatments located in the upper part. Finally, the high clay contents contribute to the increase of the capacity to retain water in the microbasin soil. Therefore, it is necessary to promote technologies that give the soil greater coverage to improve its quality and reduce the rate of water erosion.
\end{abstract}

Keywords: Moisture retention, pins erosion, steep land, dry corridor.
Recibido: 2 de octubre del 2019 Aceptado: 28 de abril del 2020
Los artículos de la revista La Calera de la Universidad Nacional Agraria, Nicaragua, se comparten bajo términos de la licencia Creative Commons: Reconocimiento, No Comercial, Compartir Igual. Las autorizaciones adicionales a las aquí delimitadas se

(C) Copyright 2020. Universidad Nacional Agraria (UNA). 


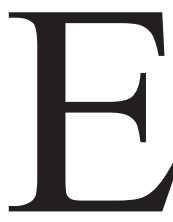
$\mathrm{n}$ un contexto de clima variable, la capacidad de transformar la agricultura para alimentar a una población creciente sin perjudicar la base de recursos naturales, no sólo permitirá alcanzar los objetivos de seguridad alimentaria, sino que también ayudará a mitigar los efectos negativos del cambio climático. Una agricultura más productiva y resistente requerirá una mejor gestión de los recursos naturales como la tierra, el agua, el suelo y los recursos genéticos, a través de buenas prácticas como la agricultura de conservación, el manejo integrado de plagas, la Agroforestería y las dietas sostenibles (Bendaña, 2012).

En la región, el uso intensivo de los suelos provoca cambios en sus propiedades llegando afectar la capacidad productiva a través de su influencia sobre la vegetación y tipos de usos posibles en la agricultura (Hernández et al., 2006). Las principales características físicas que influyen sobre la estructura del suelo son la profundidad de las raíces, régimen de humedad (capacidad de agua útil, drenaje) y del aire (macro porosidad). Estas propiedades, en iguales condiciones climáticas, son las principales causantes del cambio en la composición de la vegetación agroforestal (Lal, 2000), y junto con la actividad antropogénica provocan el surgimiento de procesos de degradación de las propiedades de los suelos (Oldeman, Van y Pulles, 1990; Kumar y Goh, 2000). Estos procesos afectan los contenidos de materia orgánica del suelo (MOS), la porosidad total $(\mathrm{Pt})$, la capacidad de campo (CC) y la humedad del suelo.

En el corredor seco de Nicaragua, Catholic Reliefe Service (CRS) está promoviendo la agricultura de conservación de suelos y agua (ACS), a través del programa Agua y suelo para la agricultura (ASA), donde se aplica los principios de rotación de cultivo, cobertura muerta, siembra directa y las 4R (Reducir, Reutilizar, Reciclar y Recuperar), como estrategia de conservación y restauración de suelos. Este sistema permite alternar plantas de diferentes familias con necesidades nutritivas diferentes en un mismo lugar durante distintos ciclos, usa la cobertura muerta, mulch o capa de material aplicada a la superficie del suelo, para conservar la humedad, mejorar la fertilidad, salud del suelo y reducir la invasión de plantas no deseadas en cultivos.

El presente estudio pretende: i) evaluar la tasa de erosión laminar en cuatro usos de la tierra a través del método de varillas; ii) identificar la relación entre la erosión laminar y las propiedades hidrofísicas en diferentes usos de la tierra, en la micro cuenca Quebrada Arriba. Todo bajo el supuesto de que los sistemas ASA restauran las propiedades hidrofísicas y conservan el suelo.

\section{MATERIALES Y MÉTODOS}

Características del área de estudio. El trabajo se realizó en la cuenca Quebrada Arriba con un área aproximada de $6.5 \mathrm{~km}^{2}$, en una elevación promedio de $806.5 \mathrm{~m}$, en la jurisdicción del municipio de Yalaguina, departamento de Madriz a 217.8 $\mathrm{km}$ del departamento de Managua, Nicaragua. Se ubica en las coordenadas $13^{\circ} 29^{\prime}$ latitud Norte y $86^{\circ} 30^{\prime}$ longitud Oeste (Figura 1). La zona presenta un clima de sabana tropical de altura (seco), la precipitación pluvial oscila entre 1000 y 1 $200 \mathrm{~mm}$, caracterizado por una buena distribución durante todo el año, la temperatura anual oscila entre 23 y $24{ }^{\circ} \mathrm{C}$ (Figura 2).

La mayor parte de los suelos de la unidad hidrográfica Quebrada Arriba está conformada por un terreno con pendientes que van desde superficies escapadas a extremadamente escarpada, y se distribuye en la parte media y alta de la cuenca, lo que favorece potenciales procesos erosivos en los sistemas tradicionales de cultivos en la zona. En cambio, los terrenos con pendiente plana constituyen el 1.2 por ciento ( 7.78 hectáreas) del área total y se concentran en la parte baja de la cuenca. Los suelos deben su origen y clasificación a la influencia combinada de factores que actúan sobre los materiales parentales depositados como son: el clima, relieve, roca madre, vegetación, organismos y el tiempo. En esta cuenca se identifican los siguientes subgrupos taxonómicos: Typic Usthorthens, Typic Argiudolls, Typic Calciusteps, Typic Haplustalfy Typic Haplusteps (Urbina y Maradiaga, 2019).

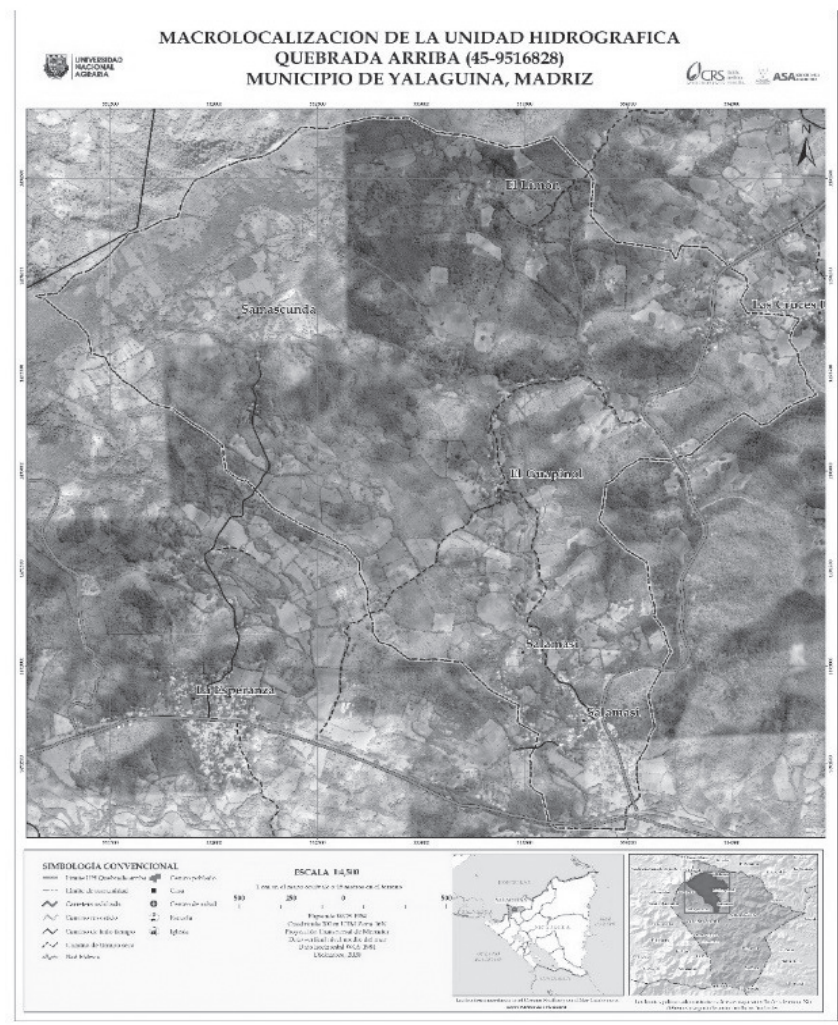

Figura 1. Ubicación del área de estudio, comunidad Quebrada Arriba, municipio de Yalagüina, Madriz, Nicaragua. 


\section{Sistemas de usos de suelo evaluados}

Sistema de coberturas de suelo (SCS). El sistema de cobertura (del programa ASA) comprende el uso de rastrojos como cobertura muerta para conservar humedad, restaurar la estructura de suelo, evitar la erosión y mejorar su calidad y biodiversidad, para proteger el medio ambiente y producir saludablemente.

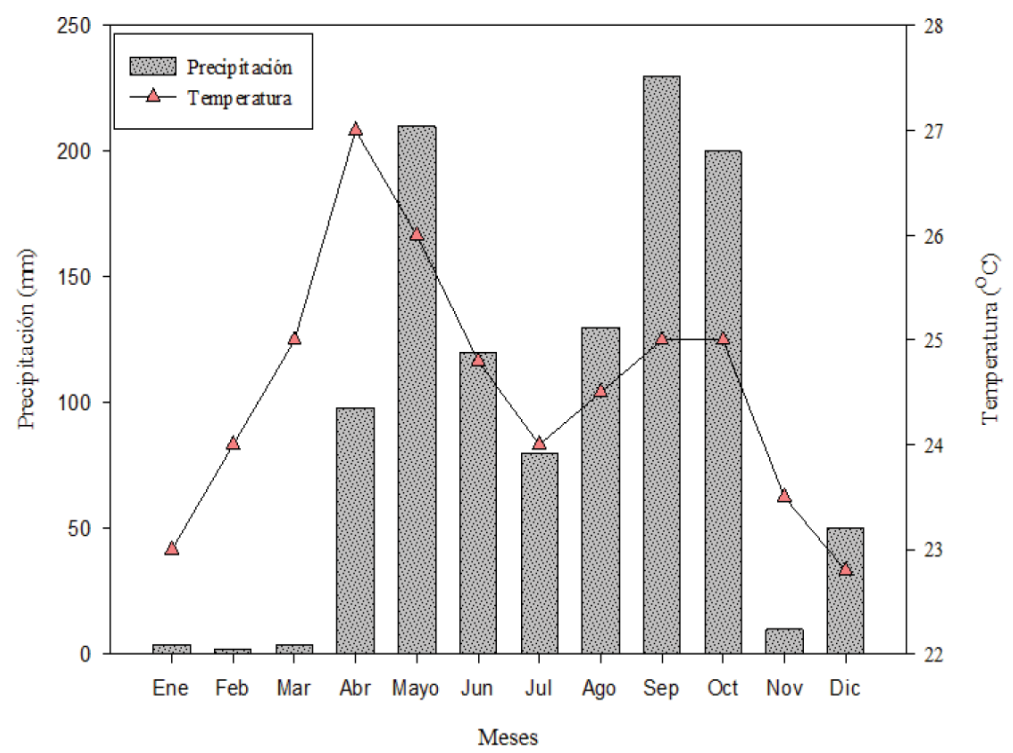

Figura 2. Comportamiento de la precipitación mensual $(\mathrm{mm})$ y la temperatura $\left({ }^{\circ} \mathrm{C}\right)$ durante el estudio. Fuente: Instituto Nicaragüense de Estudios Territoriales (INETER, 2018).

Sistema bosque secundario (Bs). Los bosques presentes en la microcuenca son clasificados como secundarios porque han sido salvados después de una tala severa para la producción agrícola.

Sistema agroforestal (SAF). El Sistema Agroforestal de árboles disperso, está conformado por plantas leñosas y perennes; son deliberadamente combinados con el cultivo en turno incorporando materia orgánica a través del rastrojo.

Sistema tradicional (Tr). Se basa en prácticas transmitidas de generación en generación, que incluye sembrar granos básicos sin el componente arbóreo, algunos queman y usan agroquímicos.

Descripción del diseño en campo. Se eligieron tres fincas en la unidad hidrográfica Quebrada Arriba, localizadas a lo largo del relieve en la parte alta, media y baja con un diseño de parcelas dividas (DPD), presentándose una unida experimental de mayor tamaño (Fincas), una parcela por sistemas de uso de la tierra (tratamiento), y en cada sistema, una unidad experimental de menor tamaño por posición en la pendiente (alta y baja). En la mayoría de las variables esta pequeña unidad fue sub dividida en dos profundidades. Estas variables se evaluaron a través del modelo: $\mathrm{Y}_{\mathrm{ijkl}}=\mu_{\mathrm{ijk} \mathrm{k}}+\beta_{\mathrm{i}}+\theta_{\mathrm{j}}$ $+\alpha_{\mathrm{k}}+\tau_{1}+\varepsilon_{(\mathrm{jik})}$. Donde: $\mathrm{i}=3$ finca; $\mathrm{j}=4$ tratamientos (descritos previamente); $\mathrm{k}=2$ posición en el relieve por parcela (alta y baja); $1=2$ profundidades; y $E$ ijk $=$ Es el error de $(\beta \theta \alpha)$ ijk.

Las técnicas estadísticas utilizadas fueron: El análisis individual por variable con un Diseño de Parcelas Divididas (DPD), y para entender el grado de asociación entre variables se aplicó un análisis de Componentes Principales (ACP). Donde se utilizaron 48 datos para los indicadores asociados a la erosión hídrica. Los análisis fueron corridos en el software (InfoStat, 2009) y graficados en el software SigmaPlot 12.0 versión libre. El modelo aplicó la separación de media de Fisher LSD, al $95 \%$ de confianza.

Registro de datos. El monitoreo se realizó en mayo del 15 al 18 y en diciembre entre los días 15 y 17 del año 2017. Se utilizó barrenos de espiral para colectar muestras a $10 \mathrm{~cm}$ de profundidad compuesta de tres sub muestras. Estas fueron colectadas en bolsas plásticas con su debida identificación en un peso de $0.5 \mathrm{~kg}$, para ser enviadas al laboratorio de suelos (LABSA), de la Universidad Nacional Agraria (UNA) en Managua. A esas muestras se les determinó; contenido de materia orgánica (método Walkley y Black, 1946), y textura del suelo (Bouyucos). Paralelamente, se tomaron muestras con cilindros de $98 \mathrm{~cm}^{3}$, para determinar en el mismo laboratorio; densidad aparente (Da) a través del método del cilindro con volumen conocido, y el porcentaje de humedad (método gravimétrico USDA). Se consideró la parte alta y baja de cada parcela, y los datos de erosión se colectaron después de ocurrido la estación lluviosa. En cada sub-parcela de erosión y por cada varilla la lectura mayor de $10 \mathrm{~cm}$ es considerada perdida (erosión), y la lectura menor de $10 \mathrm{~cm}$ es ganancia (sedimentación) de suelos respectivamente. El método de la la olla de presión de Richard fue utilizado para determinar capacidad de campo, y cilindros sencillos para medir la velocidad de infiltración de agua en el suelo.

Parcela de erosión (varillas de erosión). En cada parcela por sistema de uso se establecieron 20 varillas distribuidas de la siguiente manera: 10 varillas en la parte alta de la parcela e igual cantidad en la parte baja. Esto significa que se utilizaron un total de 80 varillas por finca y 240 a nivel de cuenca. Con una distancia promedio de 15 metros mínima entre cada cantidad aguas abajo. Cada sub parcela de 10 metros cuadrados, ubicando una varilla por metro cuadrado. 


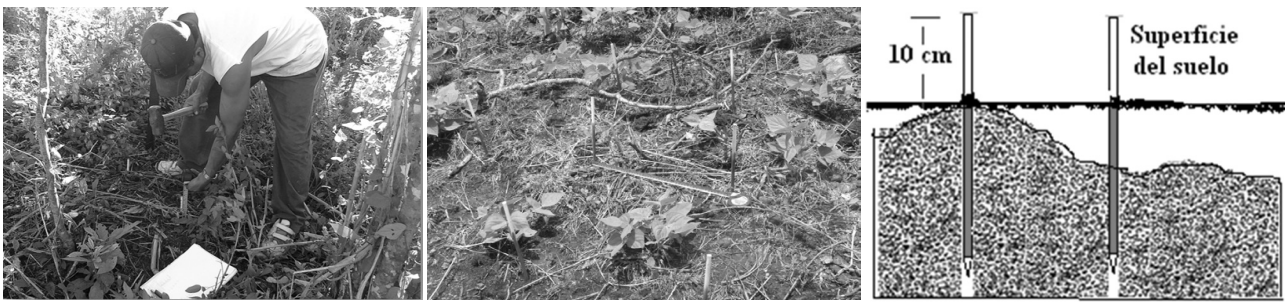

Figura 3. Instalación de varillas para el monitoreo de la erosión del suelo $(\mathrm{cm})$ con diferentes colores en las diferentes posiciones de las parcelas y la toposecuencia. Imagen tomada de Aviles et al. (2018).
Al comparar la tasa de erosión entre las varillas de cada parcela, mayor depósito de sedimentos fue observado en la parte baja (Figura 4; C) con valores de 59.81 y 96.50 ton $\mathrm{ha}^{-1}$ $\mathrm{a}^{-1}$ para la parte baja y alta respectivamente. Fenómeno asociado a la reducción de

Cada varilla de hierro liso de $3 / 8$, tenía $30 \mathrm{~cm}$ de longitud. Estas fueron enterradas $20 \mathrm{~cm}$, y pintadas para facilitar su localización durante el monitoreo. Este método fue validado por Somarriba (1989); Mendoza y Tinoco (1994); Aviles et al. (2018), en diferentes usos y manejos de suelos con pendientes diferentes. Para interpretar el riesgo de erosión laminar de pérdida de suelos, se usa la propuesta de la Organización de las Naciones Unidas para la Alimentación y la Agricultura [Organización de las Naciones Unidas para la Alimentación y la Agricultura (FAO, 1980)].

Las pérdidas de suelos son más severas en zonas de laderas, haciéndose más fuerte en las primeras etapas de los cultivos cuando el suelo está descubierto y expuesto al golpe directo de las gotas de lluvia (erosión hídrica), y a su vez destruyen las propiedades físicas del suelo (Narro, 1994). la energía gravitacional que da lugar a mayor depósito en la parte baja del relieve. Otros estudios en Nicaragua utilizando el método de varillas, en suelos volcánicos de la finca El Plantel en Tipitapa, Masaya, reportan para el cultivo de maíz sembrado en pendiente de $15 \%$ una tasa de erosión de 135.70 ton $\mathrm{ha}^{-1} \mathrm{a}^{-1}$, y en cultivo sin cobertura en una pendiente de $10 \%, 160.95$ ton $\mathrm{ha}^{-1} \mathrm{a}^{-1}$ (Somarriba, 1989). En Matagalpa, en 2005, entre los meses de julio a diciembre en la comunidad de los Alpes, municipio de San Ramón, con el método de clavos, en cultivos variados y distintos grados de pendientes, se obtuvieron tasa de erosión promedio de 102.34, 28.84, 46.48, 42 y 16.8 ton $\mathrm{ha}^{-1} \mathrm{a}^{-1}$ (Corrales, 2001).

\section{RESULTADOS Y DISCUSIÓN}

Erosión laminar. La erosión hídrica se considera la forma más importante de degradación de los suelos en el país, aproximadamente 7.7 millones de hectáreas del territorio nacional presentan grados variables de erosión, 3.6 millones de hectáreas presentan un grado de erosión catalogada de fuerte a severo (PAA-NIC, 1994). En este estudio, la tasa de erosión fue clasificada de severa para el sistema SAF y muy severa en las parcelas Bosque, SCS y Tradicional (FAO, 1980). La tasa de erosión fue significativamente diferente entre fincas (Figura $4 \mathrm{~B} ; p>\mathrm{F}=0.0005$;), con una tasa de erosión promedia de $7.55,54.15,172.77$ ton $\mathrm{ha}^{-1} \mathrm{a}^{-1}$, en las fincas de Don Federico cruz, Juan José y William Aguirre respectivamente. Probablemente, la forma y longitud del micro relieve, afecta la tasa de erosión en forma de depósito en áreas con depresiones en cada parcela experimental.Observándose en la finca de Don William, cambios abruptos de la gradiente y forma del relieve en cortas distancias, lo cual ofrece mayor detención de sedimentos en las áreas aguas abajo del micro relieve.
Infiltración de agua en el suelo. La velocidad de infiltración de agua en el suelo fue significativamente más rápida en los sistemas bosque y SAF, $(p>\mathrm{F}=0.0012)$. Los resultados fueron: $49.18,19.8,16.48$ y 14.18 pul h$^{-1}$ (equivalente a 1.22 , $3.03,3.64$, y $4.23 \mathrm{~min} \mathrm{pulg}^{-1}$ ), para los sistemas, bosque, $\mathrm{SAF}$, tradicional y CSC respectivamente. Estos resultados indican que el sistema ASA, cuenta con el tiempo necesario (3 años) para lograr niveles de agregación y formación de canales biológicos que se desarrollan en el sistema de 
bosque. Los tipos de suelo afectaron significativamente la velocidad de infiltración a nivel de finca $(p>\mathrm{F}=0.0007)$, con mayor velocidad fincas ubicadas en la parte media y alta del paisaje. Los resultados fueron: 27.03, 25.97 y 13.16 pul $\mathrm{h}^{-1}$ (equivalente a 2.22, 2.31, y 4.56 min pulg $^{-1}$, tiempos de infiltración) para la finca de Federico Cruz, Juan José y William Aguirre respectivamente. La velocidad de infiltración de agua en el suelo, también fue afectada por la posición en el relieve, con significativa mayor velocidad en la parte alta de las parcelas $(p>\mathrm{F}=0.0004)$. Los resultados fueron: 29.41 y $14.29 \mathrm{pul} \mathrm{h}^{-1}$; equivalente a 2.04 y $4.02\left(\mathrm{~min} \mathrm{pulg}^{-1}\right)$ en la parte alta y baja respectivamente. Este resultado puede estar asociado a la presencia de sedimentos finos en la parte baja de las parcelas, que han sido movilizados por la escorrentía superficial y sellando los espacios porosos de la superficie.

Contenido de materia orgánica en el suelo (MOS). En este estudio, los contenidos de materia orgánica en los suelos fueron diferentes significativamente entre sistemas de uso de la tierra, con valores promedios de 2.98, 3.95, 5.36 y $12.75 \%$, para los sistemas Tradicional, SAF, Bosque y SCS, respectivamente. Estos valores son clasificados altos en el sistema Bosques y ASA, mientras que el Tradicional y SAF son medios (Quintana, 1992). Este resultado probablemente se debe a la cantidad y tipo de cobertura que presentan estos suelos. A nivel de fincas, los contenidos de MOS tienden a aumentar en las localizadas en la parte baja de la cuenca, pero aumenta el contenido de MOS en las partes altas de las parcelas. Estos resultados podrían estar asociado de igual manera a la alta presencia de cobertura en la cabecera de los relieves, que dan lugar al arrastre de sedimento cargados de materia orgánica.

Capacidad de campo (CC). La capacidad de retener agua en suelos medida a través de la (CC), fue afectada significativamente entre sistemas de uso $(p>0.0001)$, probablemente por los contenidos de arcilla y MOS. Los valores promedios fueron de 33.57, 36.44, 38.28 y $39.76 \%$, para Bosque, Tradicional, SCS y SAF respectivamente. Según (Marrero, 2006). La capacidad de retención del agua para un suelo franco arcilloso es $30 \%$ y suelos arcillosos $38 \%$. Los valores encontrados en el sistema SAF de textura arcillosa son mayores a los reportados por Marrero (2006). La retención de humedad se vio afectada significativamente $(p>0.0001)$, con valores promedios de $41.21,33.48$ y $36.36 \%$, para las fincas de William Aguirre, Federico Cruz y Juan José respectivamente. Entre la parte alta y baja de cada parcela los valores fueron similares $(37.20$ y $36.83 \%)$. Esto es confirmado por Gliessman (2002), al plantear que cuando se incrementa los contenidos de materia orgánica en el suelo, el agua es atraída con mayor fuerza y su intensidad está determinada por el tamaño de las partículas y los contenidos de MOS.

Contenido de arcilla en el suelo. Los resultados de laboratorio de textura mostraron contenidos de arcilla ligeramente mayores en el sistema SAF, con resultados de $32.38,33.86,36.40$ y $37.46 \%$, para Bosque, Tradicional, SCS y SAF respectivamente. Sin embargo, a nivel de fincas, los contenidos de arcilla fueron significativamente mayores en la finca de Don Federico Cruz $(42.39 \%$ de arcilla), clasificada texturalmente como arcillosa. En las fincas de don Juan José y William Aguirre los contenidos fueron de $33.77 \%$ y $28.91 \%$ respectivamente, clasificados como franco arcilloso. Esto probablemente se debe a las posiciones que se encuentran los suelos en cada finca, la erosión hídrica y el tipo de material parental. En el relieve los contenidos de arcilla fueron similares entre alta y baja posición, con valores de $35.35 \%$ y $34.70 \%$ respectivamente.

Análisis multivariado. Basado en cuarenta y ocho datos para evaluar el efecto de los sistemas SCS, SAF, Bosque y Tradicional, sobre la erosión y las propiedades hidrofísicas (infiltración de agua en el suelo, capacidad de campo, contenido de arcilla, y el contenido de materia orgánica), el estudio identificó significativa y positivamente asociación entre las propiedades contenido de arcilla y la capacidad de campo ( $p>\mathrm{F}=0.0198 ; \mathrm{r} 2=0.98$ (Cuadro 1$)$.

El análisis de asociación y variabilidad de las propiedades hidrofísicas son representados en el análisis de componente principal con CP1 igual a $56.5 \%$ de la varianza (Figura 5). El cual explica que la retención de humedad asociada a los contenidos de arcilla es influenciada por los sistemas de uso con mayor cobertura vegetal, tales como los SAF y sistemas SCS; fenómeno que no ocurre en las áreas boscosas, por estar localizadas en las partes más altas del relieve con suelos superficiales y manejados con pastoreo de ganado.

Cuadro 1. Correlación múltiple entre indicadores hidrofísicas de suelo. Quebrada Arriba, Yalagüina 2017

\begin{tabular}{|c|c|c|c|c|c|}
\hline Indicadores & $\begin{array}{c}\text { Infiltración } \\
\left(\text { min pul }{ }^{-1}\right)\end{array}$ & $\begin{array}{l}\mathrm{CC} \\
(\%) \\
\end{array}$ & $\begin{array}{c}\text { Arcilla } \\
(\%)\end{array}$ & $\begin{array}{c}\text { MOS } \\
\text { (ton ha-1) }\end{array}$ & $\begin{array}{c}\mathrm{E}(-) \\
\left(\text { ton } \mathrm{ha}^{-1} \mathrm{a}^{-1}\right)\end{array}$ \\
\hline Infiltra $\left(\operatorname{min~pul}^{-1}\right)$ & 1 & & & & \\
\hline $\mathrm{CC}(\%)$ & $\begin{array}{l}0.71 \\
\text { NS }\end{array}$ & 1 & & & \\
\hline Arcilla (\%) & $\begin{array}{c}0.63 \\
\text { NS }\end{array}$ & $\begin{array}{c}0.98 \\
0.0198\end{array}$ & 1 & & \\
\hline $\operatorname{MOS}\left(\mathrm{kg} \mathrm{t}^{-1} / \mathrm{ha}^{-1}\right)$ & $\begin{array}{c}0.43 \\
\text { NS }\end{array}$ & $\begin{array}{c}0.19 \\
\text { NS }\end{array}$ & $\begin{array}{l}0.31 \\
\text { NS }\end{array}$ & 1 & \\
\hline E (-) $\left(\right.$ t ha $^{-1}$ año-1 $)$ & $\begin{array}{l}0.20 \\
\text { NS }\end{array}$ & $\begin{array}{c}-0.21 \\
\text { NS }\end{array}$ & $\begin{array}{c}-0.40 \\
\text { NS }\end{array}$ & $\begin{array}{c}-0.59 \\
\mathrm{NS}\end{array}$ & 1 \\
\hline
\end{tabular}

La fila en el valor superior representa el coeficiente de correlación " $r$ " y el valor en la parte baja la probabilidad $(p)$. 


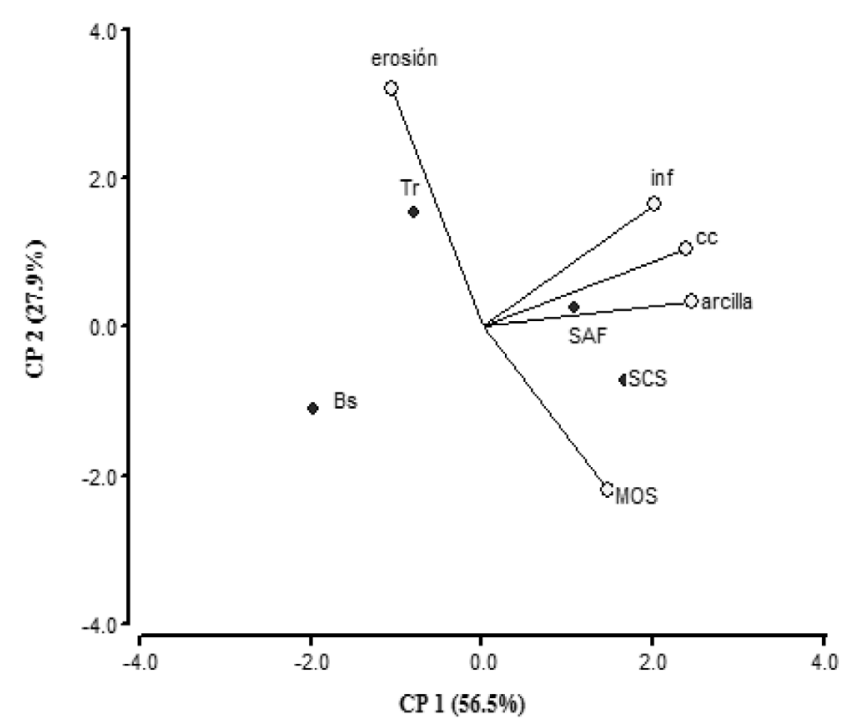

Figura 5. Análisis de componentes principales de las propiedades hidrofísicas y erosión de suelos según sistemas de uso de suelo (Bs, ASA, SAF y Tr).

Observando el análisis de asociaciones en el CP2 (27.9\% de la varianza), donde las propiedades erosión y contendido de MOS representan el mayor peso del componente, explican que el uso de la tierra con menor cobertura vegetal (sistema tradicional) es más vulnerable a ser degradado. Este análisis está basado en 48 datos de muestreo, en Yalagüina, Nicaragua, 2017.

\section{CONCLUSIONES}

Los sistemas más vulnerables a ser degradados por la erosión hídrica laminar y por pérdida de materia orgánica, son los que presentaron menor cobertura vegetal (sistema tradicional), fenómeno que confirma la necesidad de promover sistemas de cultivos con mayor cobertura de rastrojos y arbórea como los sistemas agroforestales.

Los sistemas de cultivos que ofertan mayor cobertura al suelo, presentan mayores contenidos demateria orgánica, tasa rápida de infiltración de agua en el suelo y menores tasas de erosión. Sin embargo, la retención de humedad fueasociada a los contenidos de arcilla y a su vez influenciada por los sistemas de uso con mayor cobertura vegetal, tales como los sistemas agroforestales, y de cobertura SCS. Fenómeno que no ocurrió en las áreas boscosas, por estar localizadas en las partes más altas del paisaje de la cuenca, en suelos superficiales y probablemente compactados por pastoreo de ganado.

\section{REFERENCIAS BIBLIOGRÁFICAS}

Aviles, E.A., Mendoza, R. B., Aguirre, C. Van der Hoeek, R. Mena, M., y Tellez O. (2018). Evaluación de la calidad de suelos en sistemas de cultivo-árboles-pastos, microcuenca Tecomapa, Somotillo-Nicaragua. La Calera 18(31),98 - 103.

Bendaña, G. (2012). Agua, agricultura y seguridad Alimentaria en las zonas secas de Nicaragua. 290 p.

Corrales, D. (2001). Evaluación de especies de barreras vivas sobre el control de erosión, ingresos económicos y la producción de maíz (Zea mays L.) y frijol (P. vulgaris). (Tesis de grado). Universidad Nacional Agraria. Managua, Managua.

Gliessman, S. R. (2002). Agroecología: procesos ecológicos en la agricultura sostenible. https://biowit.files.wordpress.com/2010/11/ agroecologia-procesos-ecolc3b3gicos-en-agricultura-sostenible-stephen-r-gliessman.pdf

Hernández, A., Ascanio, M., Morales, M., Bojórquez, J., García, N., y García, D. (2006). Fundamentos de la formación del suelo, cambios globales y su manejo. Editorial Universidad Autónoma de Nayarit, MX. p. 15-25.

InfoStat (2009). InfoStat, versión 2009. Manual del Usuario. Grupo InfoStat

Instituto Nicaragüense de Estudios Territoriales. (2018). Dirección General de Meteorología. Resumen Meteorológico anual. 1958 al 2020.

Kumar, K., y Goh, K. M. (2000). Crop residues and management practices: Effects on soil quality, soil nitrogen dynamic, crop yield, and nitrogen recovery. Adv. Agron. 68:197-47.

Lal, R. (2000). Physical management of soil of the tropic. Priorities for the 21st. Centuary. Soil Science, 165:191-207

Marrero, E. (2006). Unidad II De Régimen de riego. Universidad Nacional Agraria Managua, Nicaragua. 50 p.

Mendoza, M.E., y Tinoco, R. (1994). Evaluación de terraza de bordo y cultivos en curvas a nivel por el método clavos y rondanas. IRENA, SUWAR, PASOLAC. Managua, Nicaragua. p. 41.

Narro, E. (1994). Física de suelos con enfoque agrícola. Editorial México Trillas UAAAN 1994, 156 p.

Oldeman, I. R., Van, W., y Pulles, R. (1990). The extent of human induced soil degradation. ISRIC, Wageningen. The Netherlands, 34 p.

Organización de las Naciones Unidas para la Alimentación y la Agricultura. (1980). Metodología provisional para la evaluación de la degradación de los suelos. FAO-PNUMA-UNESCO. 86 p. 
PAA-NIC. (1994). Plan de Acción Ambiental de Nicaragua. MEDE, MARENA, Managua, Nicaragua.

Quintana, J. O. (1992). Manual de Fertilidad para los suelos de Nicaragua. Editorial primer territorio indígena libre de América Ithaca, Nueva York. Residencial Las Mercedes Nº 19-A. Managua, Nicaragua. 60 p.

Somarriba, M. (1989). Planificación conservacionista de la finca el plantel. (Tesis de grado). Instituto Superior de Ciencias Agropecuarias. Managua-Nicaragua. 40 p.

Urbina, G. A., y Maradiaga F, E. (2019). Evaluación del potencial biofisico para producir maíz y frijol y sorgo en la unidad hidrografica Quebrada Arriba (45-9516828), Yalagüina, Madriz, Nicaragua. (Tesis de grado). Universidad Nacional Agraria. Managua, Nicaragua. $65 \mathrm{p}$.

Walkley, A., y Black, I. A. (1946). An examination of the Digestions method for determining organic carbon in soils: Effect of variations in digestion conditions and of inorganic soil constituents. Soil Sci. 63:251-263. 